PROCEEDINGS OF THE

AMERICAN MATHEMATICAL SOCIETY

Volume 139, Number 5, May 2011, Pages 1743-1750

S 0002-9939(2010)10601-9

Article electronically published on October 18, 2010

\title{
NEW INTEGRAL IDENTITIES \\ FOR ORTHOGONAL POLYNOMIALS ON THE REAL LINE
}

\author{
D. S. LUBINSKY
}

(Communicated by Walter Van Assche)

\begin{abstract}
Let $\mu$ be a positive measure on the real line, with associated orthogonal polynomials $\left\{p_{n}\right\}$ and leading coefficients $\left\{\gamma_{n}\right\}$. Let $h \in L_{1}(\mathbb{R})$. We prove that for $n \geq 1$ and all polynomials $P$ of degree $\leq 2 n-2$,

$$
\int_{-\infty}^{\infty} \frac{P(t)}{p_{n}^{2}(t)} h\left(\frac{p_{n-1}}{p_{n}}(t)\right) d t=\frac{\gamma_{n-1}}{\gamma_{n}}\left(\int_{-\infty}^{\infty} h(t) d t\right)\left(\int P(t) d \mu(t)\right) .
$$
\end{abstract}

As a consequence, we establish weak convergence of the measures on the lefthand side.

\section{INTRODUCTION}

Let $\mu$ be a positive measure on the real line with infinitely many points in its support, and let $\int x^{j} d \mu(x)$ be finite for $j=0,1,2, \ldots$. Then we may define orthonormal polynomials

$$
p_{n}(x)=\gamma_{n} x^{n}+\cdots, \quad \gamma_{n}>0,
$$

satisfying

$$
\int_{-\infty}^{\infty} p_{n} p_{m} d \mu=\delta_{m n}
$$

Let

$$
L_{n}(x, y)=\frac{\gamma_{n-1}}{\gamma_{n}}\left(p_{n}(x) p_{n-1}(y)-p_{n-1}(x) p_{n}(y)\right)
$$

and for non-real $a$,

$$
E_{n, a}(z)=\sqrt{\frac{2 \pi}{\left|L_{n}(a, \bar{a})\right|}} L_{n}(\bar{a}, z)
$$

In a recent paper [6], we used the theory of de Branges spaces [1] to show that for $\operatorname{Im} a>0$, and all polynomials $P$ of degree $\leq 2 n-2$, we have

$$
\int_{-\infty}^{\infty} \frac{P(t)}{\left|E_{n, a}(t)\right|^{2}} d t=\int P(t) d \mu(t)
$$

Received by the editors March 23, 2010 and, in revised form, May 21, 2010.

2010 Mathematics Subject Classification. Primary 42C05.

Key words and phrases. Orthogonal polynomials on the real line, Geronimus type formula, Poisson integrals.

This research was supported by NSF grant DMS1001182 and U.S.-Israel BSF grant 2008399.

(C)2010 American Mathematical Society Reverts to public domain 28 years from publication 
This may be regarded as an analogue of Geronimus' formula for the unit circle, where instead of $E_{n, a}$, we have a multiple of the orthonormal polynomial on the unit circle in the denominator [3, Thm. V.2.2, p. 198], [8, pp. 95, 955]. There is an earlier real line analogue, due to Barry Simon [9, Theorem 2.1, p. 5], namely

$$
\frac{1}{\pi} \int_{-\infty}^{\infty} \frac{P(t)}{\left(\frac{\gamma_{n-1}}{\gamma_{n}}\right)^{2} p_{n}^{2}(t)+p_{n-1}^{2}(t)} d t=\int P(t) d \mu(t) .
$$

Simon calls this a real line orthogonal polynomial analogue of Carmona's formula and refers also to earlier work of Krutikov and Remling [5] and Carmona [2. The latter is the special case of $(1.3)$ with $\left(p_{n-1} / p_{n}\right)(\bar{a})= \pm i \gamma_{n-1} / \gamma_{n}$. In a subsequent paper, we gave a self-contained proof of (1.3), and deduced results on weak convergence, discrepancy, and Gauss quadrature.

In this paper, we first establish the following alternative form of (1.3):

Proposition 1.1. Let $\mu$ be a positive measure on the real line with infinitely many points in its support, and with $\int x^{j} d \mu(x)$ finite for $j=0,1,2, \ldots$ Let $z \in \mathbb{C} \backslash \mathbb{R}$. Then for all polynomials $P$ of degree $\leq 2 n-2$,

$$
\frac{1}{\pi}|\operatorname{Im} z| \int_{-\infty}^{\infty} \frac{P(t)}{\left|z p_{n}(t)-p_{n-1}(t)\right|^{2}} d t=\frac{\gamma_{n-1}}{\gamma_{n}} \int P(t) d \mu(t)
$$

and

$$
\frac{1}{\pi}|\operatorname{Im} z| \int_{-\infty}^{\infty} \frac{P(t)}{\left|p_{n}(t)-z p_{n-1}(t)\right|^{2}} d t=\frac{\gamma_{n-1}}{\gamma_{n}} \int P(t) d \mu(t) .
$$

The factor involving $z$ inside the integral above is essentially the Poisson kernel for the upper-half plane. By using limiting properties of Poisson integrals, we deduce our main result, a new integral identity for orthogonal polynomials:

Theorem 1.2. Let $\mu$ be a positive measure on the real line with infinitely many points in its support, and with $\int x^{j} d \mu(x)$ finite for $j=0,1,2, \ldots$ Let $\left\{p_{n}\right\}$ and $\left\{\gamma_{n}\right\}$ denote, respectively, the orthogonal polynomials, and leading coefficients corresponding to $\mu$. Let $h \in L_{1}(\mathbb{R})$. Then for all polynomials $P$ of degree $\leq 2 n-2$,

$$
\int_{-\infty}^{\infty} \frac{P(t)}{p_{n}(t)^{2}} h\left(\frac{p_{n-1}(t)}{p_{n}(t)}\right) d t=\frac{\gamma_{n-1}}{\gamma_{n}}\left(\int_{-\infty}^{\infty} h(t) d t\right)\left(\int P(t) d \mu(t)\right)
$$

and

$$
\int_{-\infty}^{\infty} \frac{P(t)}{p_{n-1}(t)^{2}} h\left(\frac{p_{n}(t)}{p_{n-1}(t)}\right) d t=\frac{\gamma_{n-1}}{\gamma_{n}}\left(\int_{-\infty}^{\infty} h(t) d t\right)\left(\int P(t) d \mu(t)\right) .
$$

Note that if we choose $P=p_{n-1}^{2}$ in (1.7), we obtain, if the denominator integral is not 0 ,

$$
\frac{\gamma_{n-1}}{\gamma_{n}}=\frac{\int_{-\infty}^{\infty} h\left(\frac{p_{n}(t)}{p_{n-1}(t)}\right) d t}{\int_{-\infty}^{\infty} h(t) d t} .
$$

It might be possible to derive this special case in an alternative way, i.e., from the partial fraction expansion of $\frac{p_{n-1}}{p_{n}}(x)$ and known formulae for the distribution function, meas $\left\{x: \frac{p_{n-1}}{p_{n}}(x)>t\right\}$. We may replace $h(t) d t$ in (1.6) and (1.7) by a signed measure $d \nu(t)$ of finite total mass, provided one appropriately defines 
$d \nu\left(\frac{p_{n}(t)}{p_{n-1}(t)}\right)$ over each interval in which $\frac{p_{n}(t)}{p_{n-1}(t)}$ is monotone. If we choose $h(x)=$ $\frac{\log x^{-2}}{1-x^{2}}$, in Theorem 1.2, we obtain an entropy-type integral:

Corollary 1.3. With the notation of Theorem 1.2,

$$
\frac{2}{\pi^{2}} \int_{-\infty}^{\infty} P(t) \frac{\ln \left|p_{n-1}(t)\right|-\ln \left|p_{n}(t)\right|}{p_{n-1}(t)^{2}-p_{n}(t)^{2}} d t=\frac{\gamma_{n-1}}{\gamma_{n}} \int P(t) d \mu(t) .
$$

We also obtain a weak convergence-type result: recall that $\mu$ is said to be determinate if the moment problem

$$
\int x^{j} d \nu(x)=\int x^{j} d \mu(x), \quad j=0,1,2, \ldots,
$$

has the unique solution $\nu=\mu$ from the class of positive measures. We also say that a function $f$ has polynomial growth at $\infty$ if for some $L>0$ and for large enough $|x|$

$$
|f(x)| \leq|x|^{L}
$$

Theorem 1.4. Assume the hypotheses of Theorem 1.2, and in addition assume that $\mu$ is determinate. Then for all functions $f: \mathbb{R} \rightarrow \mathbb{R}$ having polynomial growth at $\infty$, and such that they are Riemann-Stieltjes integrable with respect to $\mu$, we have

$$
\begin{gathered}
\lim _{n \rightarrow \infty}\left(\frac{\gamma_{n-1}}{\gamma_{n}}\right)^{-1} \int_{-\infty}^{\infty} \frac{f(t)}{p_{n}(t)^{2}} h\left(\frac{p_{n-1}(t)}{p_{n}(t)}\right) d t \\
=\left(\int_{-\infty}^{\infty} h(t) d t\right)\left(\int f(t) d \mu(t)\right)
\end{gathered}
$$

and

$$
\begin{gathered}
\lim _{n \rightarrow \infty}\left(\frac{\gamma_{n-1}}{\gamma_{n}}\right)^{-1} \int_{-\infty}^{\infty} \frac{f(t)}{p_{n-1}(t)^{2}} h\left(\frac{p_{n}(t)}{p_{n-1}(t)}\right) d t \\
=\left(\int_{-\infty}^{\infty} h(t) d t\right)\left(\int f(t) d \mu(t)\right) .
\end{gathered}
$$

Of course, if $f$ is continuous on the real line, it will be locally Riemann-Stieltjes integrable with respect to $\mu$. Simon 9] proved weak convergence involving his Carmona-type formula.

\section{Proof of the Results}

Proof of Proposition 1.1. Fix $z \in \mathbb{C} \backslash \mathbb{R}$. Choose $a \in \mathbb{C}$ such that

$$
p_{n-1}(\bar{a})=z p_{n}(\bar{a}) \text {. }
$$

There are $n$ choices for $a$, counting multiplicity. Then from (1.1), we see that

$$
L_{n}(\bar{a}, t)=-\frac{\gamma_{n-1}}{\gamma_{n}} p_{n}(\bar{a})\left(z p_{n}(t)-p_{n-1}(t)\right)
$$

and

$$
L_{n}(a, \bar{a})=2 i \frac{\gamma_{n-1}}{\gamma_{n}} \operatorname{Im}(z)\left|p_{n}(a)\right|^{2}
$$


Hence

$$
\begin{aligned}
\left|E_{n, a}(t)\right|^{2} & =\frac{2 \pi}{\left|L_{n}(a, \bar{a})\right|}\left|L_{n}(\bar{a}, t)\right|^{2} \\
& =\frac{\pi}{|\operatorname{Im} z|} \frac{\gamma_{n-1}}{\gamma_{n}}\left|z p_{n}(t)-p_{n-1}(t)\right|^{2} .
\end{aligned}
$$

Substituting into (1.3) gives (1.4), while replacing $z$ by $\frac{1}{z}$ in (1.4) gives (1.5).

Proof of (1.6) of Theorem 1.2.

Step 1: A Poisson integral identity. Let $z=x+i y$, where $y>0$. We can recast (1.4) as

$$
\int_{-\infty}^{\infty} P(t) \frac{1}{\pi} \frac{y}{\left(p_{n}(t) x-p_{n-1}(t)\right)^{2}+y^{2} p_{n}^{2}(t)} d t=\frac{\gamma_{n-1}}{\gamma_{n}} \int P(t) d \mu(t) .
$$

Let $h \in L_{1}(\mathbb{R})$. We multiply $(2.1)$ by $h(x)$, integrate over the real line, and interchange integrals, obtaining

$$
\begin{aligned}
& \int_{-\infty}^{\infty} P(t)\left[\frac{1}{\pi} \int_{-\infty}^{\infty} \frac{y h(x)}{\left(p_{n}(t) x-p_{n-1}(t)\right)^{2}+y^{2} p_{n}^{2}(t)} d x\right] d t \\
& =\frac{\gamma_{n-1}}{\gamma_{n}}\left(\int_{-\infty}^{\infty} h(t) d t\right)\left(\int P(t) d \mu(t)\right) .
\end{aligned}
$$

This is justified if the integral on the left converges absolutely, namely,

$$
\int_{-\infty}^{\infty}\left[\int_{-\infty}^{\infty} \frac{|P(t)||h(x)|}{\left(p_{n}(t) x-p_{n-1}(t)\right)^{2}+y^{2} p_{n}^{2}(t)} d x\right] d t<\infty .
$$

To prove this, choose $A$ such that all zeros of $p_{n}$ lie in $(-A, A)$. Let

$$
c=\inf _{t, x \in \mathbb{R}}\left[\left(p_{n}(t) x-p_{n-1}(t)\right)^{2}+y^{2} p_{n}^{2}(t)\right] .
$$

This is positive, as $p_{n-1}$ and $p_{n}$ do not have common zeros. Then we can bound the left-hand side in (2.3) above by

$$
\int_{|t| \geq A} \frac{|P(t)|}{y^{2} p_{n}^{2}(t)}\left(\int_{-\infty}^{\infty}|h(x)| d x\right) d t+\int_{|t| \leq A}|P(t)|\left(\int_{-\infty}^{\infty}|h(x)| d x\right) d t / c<\infty .
$$

Thus (2.3) is valid. Recall that if $h \in L_{1}(\mathbb{R})$, its Poisson integral for the upper-half plane is

$$
\mathcal{P}[h](\alpha+i \beta)=\frac{1}{\pi} \int_{-\infty}^{\infty} \frac{\beta}{(x-\alpha)^{2}+\beta^{2}} h(x) d x .
$$

We can recast $(2.2)$ as

$$
\int_{-\infty}^{\infty} \frac{P(t)}{p_{n}^{2}(t)} \mathcal{P}[h]\left(\frac{p_{n-1}(t)}{p_{n}(t)}+i y\right) d t=\frac{\gamma_{n-1}}{\gamma_{n}}\left(\int_{-\infty}^{\infty} h(t) d t\right)\left(\int P(t) d \mu(t)\right) .
$$

Step 2: The case where $h$ is bounded and has compact support. Firstly, as $h$ is bounded, we have the elementary bound

$$
\left|\mathcal{P}[h]\left(\frac{p_{n-1}(t)}{p_{n}(t)}+i y\right)\right| \leq\|h\|_{L_{\infty}(\mathbb{R})},
$$


valid for all $y$ and $t$. Next, if $\frac{p_{n-1}(t)}{p_{n}(t)}$ is a Lebesgue point of $h$, we have the classic result

$$
\lim _{y \rightarrow 0+} \mathcal{P}[h]\left(\frac{p_{n-1}(t)}{p_{n}(t)}+i y\right)=h\left(\frac{p_{n-1}(t)}{p_{n}(t)}\right) .
$$

Now, if $u$ is not a Lebesgue point of $h$ (and such points have measure 0 ), the equation $\frac{p_{n-1}(t)}{p_{n}(t)}=u$ has at most $n$ solutions for $t$, and locally these vary differentiably with $u$. It follows that (2.5) holds for a.e. $t$.

Let $\varepsilon>0$ and $\mathcal{E}_{\varepsilon}$ denote the union of $n$ closed intervals of radius $\varepsilon$, centered on the zeros of $p_{n}$. Since $P(t) / p_{n}^{2}(t)=O\left(t^{-2}\right)$ at $\infty$, we may use Lebesgue's Dominated Convergence Theorem to deduce that

$$
\begin{gathered}
\lim _{y \rightarrow 0+} \int_{\mathbb{R} \backslash \mathcal{E}_{\varepsilon}} \frac{P(t)}{p_{n}^{2}(t)} \mathcal{P}[h]\left(\frac{p_{n-1}(t)}{p_{n}(t)}+i y\right) d t \\
=\int_{\mathbb{R} \backslash \mathcal{E}_{\varepsilon}} \frac{P(t)}{p_{n}^{2}(t)} h\left(\frac{p_{n-1}(t)}{p_{n}(t)}\right) d t .
\end{gathered}
$$

It remains to estimate

$$
I_{\varepsilon, y}=\int_{\mathcal{E}_{\varepsilon}} \frac{P(t)}{p_{n}^{2}(t)} \mathcal{P}[h]\left(\frac{p_{n-1}(t)}{p_{n}(t)}+i y\right) d t
$$

and

$$
I_{\varepsilon, 0}=\int_{\mathcal{E}_{\varepsilon}} \frac{P(t)}{p_{n}^{2}(t)} h\left(\frac{p_{n-1}(t)}{p_{n}(t)}\right) d t .
$$

As $p_{n-1}$ and $p_{n}$ have no common zeros, if $\varepsilon>0$ is small enough,

$$
\inf _{\mathcal{E}_{\varepsilon}}\left|p_{n-1}\right|>0 .
$$

Moreover, as $h$ has compact support, we may choose $\varepsilon>0$ so small that for $x$ in the support of $h$ and $t \in \mathcal{E}_{\varepsilon}$, we have

$$
\left|p_{n}(t) x-p_{n-1}(t)\right| \geq \frac{1}{2}\left|p_{n-1}(t)\right| .
$$

Then for $0<y \leq 1$

$$
\begin{aligned}
\left|I_{\varepsilon, y}\right| & =\left|\frac{y}{\pi} \int_{\mathcal{E}_{\varepsilon}}\left[\int_{-\infty}^{\infty} \frac{P(t) h(x)}{\left(p_{n}(t) x-p_{n-1}(t)\right)^{2}+y^{2} p_{n}^{2}(t)} d x\right] d t\right| \\
& \leq \frac{1}{\pi} \int_{\mathcal{E}_{\varepsilon}}\left[\int_{-\infty}^{\infty} \frac{|P(t)||h(x)|}{\left(\frac{1}{2}\left|p_{n-1}(t)\right|\right)^{2}} d x\right] d t \\
& \leq \frac{4}{\pi} \sup _{t \in \mathcal{E}_{\varepsilon}}\left|\frac{P(t)}{p_{n-1}^{2}(t)}\right|\left(\int_{-\infty}^{\infty}|h(x)| d x\right) \int_{\mathcal{E}_{\varepsilon}} 1 d t .
\end{aligned}
$$

This is a bound independent of $y$ and decreases to 0 as $\varepsilon$ decreases to 0 . Finally, if $\varepsilon>0$ is small enough, $h\left(\frac{p_{n-1}(t)}{p_{n}(t)}\right)=0$ for $t \in \mathcal{E}_{\varepsilon}$ (recall that $h$ has compact support), so for such an $\varepsilon$,

$$
I_{\varepsilon, 0}=0 .
$$

Combining the above, we obtain

$$
\lim _{y \rightarrow 0+} \int_{-\infty}^{\infty} \frac{P(t)}{p_{n}^{2}(t)} \mathcal{P}[h]\left(\frac{p_{n-1}(t)}{p_{n}(t)}+i y\right) d t=\int_{-\infty}^{\infty} \frac{P(t)}{p_{n}^{2}(t)} h\left(\frac{p_{n-1}(t)}{p_{n}(t)}\right) d t
$$


and hence, from (2.4),

$$
\int_{-\infty}^{\infty} \frac{P(t)}{p_{n}^{2}(t)} h\left(\frac{p_{n-1}(t)}{p_{n}(t)}\right) d t=\frac{\gamma_{n-1}}{\gamma_{n}}\left(\int_{-\infty}^{\infty} h(t) d t\right)\left(\int P(t) d \mu(t)\right) .
$$

Thus we have (1.6) for the case where $h$ is bounded and has compact support.

Step 3: The case where $h$ is bounded but has non-compact support. Let

$$
h_{m}=h \chi_{[-m, m]}, \quad m \geq 1 .
$$

We have (1.6) for $h_{m}$; that is,

$$
\frac{1}{\pi} \int_{-\infty}^{\infty} \frac{P(t)}{p_{n}(t)^{2}} h_{m}\left(\frac{p_{n-1}(t)}{p_{n}(t)}\right) d t=\frac{\gamma_{n-1}}{\gamma_{n}}\left(\int_{-\infty}^{\infty} h_{m}\right) \int P d \mu .
$$

Now for each $t$ with $p_{n}(t) \neq 0$ and all large enough $m$,

$$
h_{m}\left(\frac{p_{n-1}(t)}{p_{n}(t)}\right)=h\left(\frac{p_{n-1}(t)}{p_{n}(t)}\right) .
$$

Next,

$$
\left|\frac{P(t)}{p_{n}(t)^{2}} h_{m}\left(\frac{p_{n-1}(t)}{p_{n}(t)}\right)\right| \leq\left|\frac{P(t)}{p_{n}(t)^{2}} h\left(\frac{p_{n-1}(t)}{p_{n}(t)}\right)\right| .
$$

This upper bound is independent of $m$ and moreover is integrable over $(-\infty, \infty)$, since it is $O\left(t^{-2}\right)$ at $\infty$ and has an integrable singularity at each zero of $p_{n}$. To see the latter, we proceed as follows. Let $x_{j n}$ be a zero of $p_{n}$. We can write, in $\left(x_{j n}, x_{j n}+\varepsilon\right]$, with small enough $\varepsilon>0$,

$$
\frac{p_{n-1}(t)}{p_{n}(t)}=\frac{g(t)}{t-x_{j n}}
$$

where $g$ is non-vanishing and continuously differentiable. If $\varepsilon>0$ is small enough, we have for some appropriate constant $C$ and $t \in\left(x_{j n}, x_{j n}+\varepsilon\right]$ :

$$
\begin{aligned}
\left|\frac{P(t)}{p_{n}(t)^{2}} h\left(\frac{p_{n-1}(t)}{p_{n}(t)}\right)\right| & \leq C \frac{1}{\left(t-x_{j n}\right)^{2}}\left|h\left(\frac{g(t)}{t-x_{j n}}\right)\right| \\
& \leq C\left|\frac{g^{\prime}(t)\left(t-x_{j n}\right)-g(t)}{\left(t-x_{j n}\right)^{2}}\right|\left|h\left(\frac{g(t)}{t-x_{j n}}\right)\right| \\
& =C\left|\frac{d}{d t}\left(\frac{g(t)}{t-x_{j n}}\right)\right|\left|h\left(\frac{g(t)}{t-x_{j n}}\right)\right| .
\end{aligned}
$$

In the second to last line, we use the fact that if $\varepsilon$ is small enough, $|g(t)| \gg$ $\left|g^{\prime}(t)\left(t-x_{j n}\right)\right|$, while $|g|$ is bounded below. Then, if $g\left(x_{j n}\right)>0$, the substitution $s=\frac{g(t)}{t-x_{j n}}$ gives

$$
\begin{aligned}
\int_{x_{j n}}^{x_{j n}+\varepsilon}\left|\frac{P(t)}{p_{n}(t)^{2}} h\left(\frac{p_{n-1}(t)}{p_{n}(t)}\right)\right| d t & \leq C \int_{x_{j n}}^{x_{j n}+\varepsilon}\left|h\left(\frac{g(t)}{t-x_{j n}}\right)\right|\left|\frac{d}{d t}\left(\frac{g(t)}{t-x_{j n}}\right)\right| d t \\
& =C \int_{\frac{g\left(x_{j n}+\varepsilon\right)}{\varepsilon}}^{\infty}|h(s)| d s \leq C \int_{-\infty}^{\infty}|h(s)| d s .
\end{aligned}
$$


If $g\left(x_{j n}\right)<0$, we proceed similarly. Thus, indeed, the function $\left|\frac{P(t)}{p_{n}(t)^{2}} h\left(\frac{p_{n-1}(t)}{p_{n}(t)}\right)\right|$ provides an integrable bound independent of $m$. Then Lebesgue's Dominated Convergence Theorem allows us to let $m \rightarrow \infty$ in (2.9) to obtain (1.6) for the case where $h$ is bounded but has non-compact support.

Step 4: The case where $h$ is unbounded. Let us define

$$
H_{m}(t)=\left\{\begin{array}{cc}
h(t), & \text { if }|h(t)| \leq m \\
0, & \text { otherwise }
\end{array}\right.
$$

We have that (1.6) holds for $h=H_{m}$. Next, for each $t$ with $p_{n}(t) \neq 0, h\left(\frac{p_{n-1}(t)}{p_{n}(t)}\right)$ finite, and all large enough $m$,

$$
H_{m}\left(\frac{p_{n-1}(t)}{p_{n}(t)}\right)=h\left(\frac{p_{n-1}(t)}{p_{n}(t)}\right) .
$$

Moreover, $\left|\frac{P(t)}{p_{n}(t)^{2}} H_{m}\left(\frac{p_{n-1}(t)}{p_{n}(t)}\right)\right|$ admits the same integrable bound as in Step 3 . Then Lebesgue's Dominated Convergence Theorem gives the result.

Proof of (1.7) of Theorem 1.2. For the given $h$, define a new function $\tilde{h}$ by

$$
\tilde{h}(x)=x^{-2} h\left(x^{-1}\right) .
$$

A substitution shows that also $\tilde{h} \in L_{1}(\mathbb{R})$ and

$$
\frac{1}{p_{n}^{2}(t)} \tilde{h}\left(\frac{p_{n-1}(t)}{p_{n}(t)}\right)=\frac{1}{p_{n-1}^{2}(t)} h\left(\frac{p_{n}(t)}{p_{n-1}(t)}\right) .
$$

So applying (1.6) to $\tilde{h}$ gives (1.7) for $h$.

Proof of Corollary 1.3. Choose in (1.6) of Theorem 1.2

$$
h(x)=\frac{\log x^{-2}}{1-x^{2}},
$$

which has $h \in L_{1}(\mathbb{R})$. Moreover, the fact that $h$ is even and a substitution show that [4, p. 533, 4.231.13]

$$
\int_{-\infty}^{\infty} h=8 \int_{0}^{1} \frac{\log x^{-1}}{1-x^{2}} d x=\pi^{2} .
$$

Proof of Theorem 1.4. We may prove the result for non-negative $h$, because every $h$ satisfying the hypotheses of Theorem 1.2 is the difference of two non-negative functions satisfying the same hypotheses. Let $f$ be Riemann-Stieltjes integrable with respect to $\mu$ and of polynomial growth at $\infty$, and let $\varepsilon>0$. Since $\mu$ is determinate, there exist upper and lower polynomials $P_{u}$ and $P_{\ell}$ such that

$$
P_{\ell} \leq f \leq P_{u} \quad \text { in }(-\infty, \infty)
$$

and

$$
\int\left(P_{u}-P_{\ell}\right) d \mu<\varepsilon
$$


See, for example, [3, Theorem 3.3, p. 73]. Then for $n$ so large that $2 n-2$ exceeds the degree of $P_{u}$ and $P_{\ell},(1.3)$ gives

$$
\begin{aligned}
& \left(\frac{\gamma_{n-1}}{\gamma_{n}}\right)^{-1} \int_{-\infty}^{\infty} \frac{f}{p_{n-1}^{2}} h\left(\frac{p_{n}}{p_{n-1}}\right)-\int f d \mu \\
& \quad=\left(\frac{\gamma_{n-1}}{\gamma_{n}}\right)^{-1} \int_{-\infty}^{\infty} \frac{f-P_{\ell}}{p_{n-1}^{2}} h\left(\frac{p_{n}}{p_{n-1}}\right)-\int\left(f-P_{\ell}\right) d \mu \\
& \leq\left(\frac{\gamma_{n-1}}{\gamma_{n}}\right)^{-1} \int_{-\infty}^{\infty} \frac{P_{u}-P_{\ell}}{p_{n-1}^{2}} h\left(\frac{p_{n}}{p_{n-1}}\right)-0 \\
& \quad=\int\left(P_{u}-P_{\ell}\right) d \mu<\varepsilon .
\end{aligned}
$$

Similarly, for large enough $n$,

$$
\begin{aligned}
& \left(\frac{\gamma_{n-1}}{\gamma_{n}}\right)^{-1} \int_{-\infty}^{\infty} \frac{f}{p_{n-1}^{2}} h\left(\frac{p_{n}}{p_{n-1}}\right)-\int f d \mu \\
& \quad=\left(\frac{\gamma_{n-1}}{\gamma_{n}}\right)^{-1} \int_{-\infty}^{\infty} \frac{f-P_{u}}{p_{n-1}^{2}} h\left(\frac{p_{n}}{p_{n-1}}\right)-\int\left(f-P_{u}\right) d \mu \\
& \quad \geq\left(\frac{\gamma_{n-1}}{\gamma_{n}}\right)^{-1} \int_{-\infty}^{\infty} \frac{P_{\ell}-P_{u}}{p_{n-1}^{2}} h\left(\frac{p_{n}}{p_{n-1}}\right)-0 \\
& \quad=\int\left(P_{\ell}-P_{u}\right) d \mu>-\varepsilon .
\end{aligned}
$$

\section{REFERENCES}

[1] L. de Branges, Hilbert Spaces of Entire Functions, Prentice Hall, Englewood Cliffs, New Jersey, 1968. MR0229011 (37:4590)

[2] R. Carmona, One-dimensional Schrödinger operators with random or deterministic potentials: New spectral types, J. Funct. Anal. 51 (1983), 229-258. MR701057 (85k:34144)

[3] G. Freud, Orthogonal Polynomials, Pergamon Press/Akademiai Kiado, Budapest, 1971.

[4] I. S. Gradshteyn and I. M. Ryzhik, Tables of Integrals, Series, and Products, Academic Press, New York, 1980. MR0582453 (81g:33001)

[5] Denis Krutikov and C. Remling, Schrödinger operators with sparse potentials: asymptotics of the Fourier transform of the spectral measure, Comm. Math. Phys. 223 (2001), 509-532. MR.1866165 (2002k:81064)

[6] D. S. Lubinsky, Universality Limits for Random Matrices and de Branges Spaces of Entire Functions, Journal of Functional Analysis 256 (2009), 3688-3729. MR2514057

[7] D. S. Lubinsky, Applications of New Geronimus Type Identities for Real Orthogonal Polynomials, Proc. Amer. Math. Soc. 138 (2010), 2125-2134.

[8] B. Simon, Orthogonal Polynomials on the Unit Circle, Parts 1 and 2, American Mathematical Society, Providence, RI, 2005. MR2105088(2006a:42002a)

[9] B. Simon, Orthogonal Polynomials with Exponentially Decaying Recursion Coefficients, in Probability and Mathematical Physics (eds. D. Dawson, V. Jaksic and B. Vainberg), CRM Proc. and Lecture Notes, 42, Amer. Math. Soc., Providence, RI, 2007, 453-463. MR.2352283 (2008k:42087)

School of Mathematics, Georgia Institute of Technology, Atlanta, Georgia $30332-$ 0160

E-mail address: lubinsky@math.gatech.edu 